\title{
A longitudinal pilot proton MRS investigation of the manic and euthymic states of bipolar disorder
}

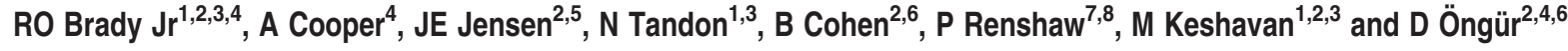

Several lines of evidence implicate dysfunction in brain energy production as a key component of bipolar disorder. In particular, elevated brain lactate levels observed in this condition suggest a shift from aerobic to anaerobic metabolism, possibly as a result of mitochondrial abnormalities. Most prior imaging studies of brain metabolites were performed in either euthymic or depressed bipolar patients or compared different populations in different mood states. We sought to measure brain metabolite concentrations in the same patients in both manic and euthymic states. Given the dramatic changes in clinical state of bipolar disorder patients, we hypothesized that previously observed abnormalities in lactate concentrations in bipolar disorder might show state dependent changes. In this study 15 patients (mean age 36.1 years) diagnosed with bipolar I disorder underwent proton magnetic resonance spectroscopy of the anterior cingulate cortex and parieto-occipital cortex during hospitalization for acute mania (mean Young Mania Rating Scale (YMRS) 22.1). Seven of these subjects returned (mean interval 21.16 months) to have imaging repeated while euthymic (mean YMRS 2.0). A group of age- and gender-matched control participants $(N=6)$ were scanned as well. We report that during mania, bipolar disorder subjects had lactate levels comparable to healthy control subjects but during euthymia these levels were significantly reduced. No significant change was observed for other metabolites. These results implicate mood dependent alterations in energy metabolism in the biology of bipolar disorder. Additionally, this finding has potential use as a biomarker for both evaluating novel treatments as well as diagnostic clarification between mood disorders.

Translational Psychiatry (2012) 2, e160; doi:10.1038/tp.2012.84; published online 11 September 2012

\section{Introduction}

Multiple lines of evidence suggest that metabolic dysfunction is a key feature of bipolar disorder (see Stork and Renshaw $^{1}$ and Dager et al. ${ }^{2}$ for review). In particular, diseasespecific abnormalities of mitochondria and energy production have been observed in bipolar disorder. ${ }^{3-5}$ Although these investigations have accumulated evidence linking metabolic dysfunction to the bipolar disorder trait, the role of metabolic dysfunction in switches between clinical states of depression, mania and euthymia has never been well characterized.

Prior studies have compared different mood states in separate populations of patients with a bipolar disorder diagnosis. These cross-sectional studies use a design, which compares populations whose clinical and treatment histories and prognosis vary widely. Prior longitudinal studies demonstrated that patients who present with mania and receive a bipolar disorder diagnosis will have variable clinical courses with different levels of inter-episode impairment, widely varying temporal courses and possibly very different eventual diagnoses. ${ }^{6,7}$ Clearly, a longitudinal study design with intrasubject measures of the same patients in different mood states would allow better characterization of state related physiological changes.

Few prior studies of brain metabolism in bipolar disorder have utilized high field magnetic resonance spectroscopy (MRS), which allows improved characterization of in vivo neurochemistry over older techniques. In this 4T Proton MRS study, we measured brain metabolite concentrations, including lactate, a measure related to energy metabolism, in the anterior cingulate cortex (ACC) and parietal occipital cortex (POC) in a cohort of medicated patients with a diagnosis of bipolar disorder type I. We chose the ACC because it is a region strongly implicated in the pathophysiology of bipolar disorder. ${ }^{8}$ We chose the POC as the comparison region. To increase the power of our study and minimize medication confounds, we performed our assessments in a single cohort of patients when they were clinically manic and in the same patients when they were clinically euthymic. An age and gender matched set of healthy control $(\mathrm{HC})$ subjects were also scanned using the same protocol. We hypothesized that given the dramatic changes in clinical state of bipolar disorder patients, abnormalities in markers of brain metabolism previously observed in bipolar disorder might show state dependent changes.

\footnotetext{
${ }^{1}$ Department of Psychiatry, Beth-Israel Deaconess Medical Center, Boston, MA, USA; ${ }^{2}$ Department of Psychiatry, Harvard Medical School, Boston, MA, USA; ${ }^{3}$ Department of Psychiatry, Massachusetts Mental Health Center, Boston, MA, USA; ${ }^{4}$ Psychotic Disorders Division, McLean Hospital, Belmont, MA, USA; ${ }^{5}$ Brain Imaging Center, McLean Hospital, Belmont, MA, USA; ${ }^{6}$ Shervert Frazier Research Institute, McLean Hospital, Belmont, MA, USA; ${ }^{7}$ Brain Institute, University of Utah, Salt Lake City, UT, USA and ${ }^{8}$ Department of Psychiatry, University of Utah School of Medicine, Salt Lake City, UT, USA

Correspondence: Dr RO Brady Jr, Department of Psychiatry, Massachusetts Mental Health Center, 75 Fenwood Road Ste 616, Boston, MA 02115, USA.

E-mail: robrady@bidmc.harvard.edu

Keywords: anterior cingulate cortex; bipolar disorder; lactate; mitochondria; MRS

Received 25 June 2012; accepted 5 July 2012
} 


\section{Materials and methods}

Subjects. Subject recruitment and proton MRS imaging were performed at the McLean Hospital, (Belmont, MA, USA) as previously described. ${ }^{9}$ Following approval by the Institutional Review Board of McLean Hospital, 39 subjects diagnosed with bipolar I disorder in a current manic state as per DSM-IV were recruited from a psychiatric inpatient unit. Twenty-two HC subjects were recruited concurrently. Diagnoses were determined by consensus based on structured clinical interviews for DSM disorders ${ }^{10}$ by a trained clinician, and all available clinical data. Subjects with significant neurological or medical problems, current substance abuse, or history of substance dependence were excluded. Tobacco smokers were not excluded from the study. All patients had negative urine toxicology tests at the time of scanning. Patients were assessed using the Young Mania Rating Scale (YMRS), Positive And Negative Syndrome Scale (PANSS) and Montgomery-Asberg depression rating scale (MADRS) on scan days. Current medication regimens were recorded and chlorpromazine (CPZ) equivalents were calculated for patients taking antipsychotic medications. HC subjects were assessed using the structured clinical interviews for DSM disorders to rule out axis I disorders. None of the patients met criteria for rapid cycling. Usable data were obtained from $21 \mathrm{HC}$ and $15 \mathrm{BD}$ subjects. Twenty-two patients were unable to tolerate the scanning environment because of their psychiatric condition and were therefore not included in the study. Two other patients elected to enter the study but refused the scan after structured clinical interviews for DSM disorders and symptoms scales were collected. The bipolar I disorder subjects who did not complete the study were more symptomatic than those who completed the study (non-completer average YMRS: 26.9; MADRS: 18.4; PANSS: 82.0; completer average YMRS: 24.7, MADRS: 11.1, PANSS: 59.6). Patients with bipolar disorder in a manic phase who completed the 4T MRS scan and symptom scales were subsequently contacted (between 12-50 months later) for follow-up assessments. These patients were re-assessed with the same exclusion criteria described above. At follow-up, all were clinically euthymic and none met DSM-IV criteria for mania, hypomania or major depressive episode. Although symptomatology score cutoffs were not used to define euthymia for the purposes of inclusion, the scores recorded at the time of the second scan suggest subjects were no more than minimally symptomatic (Table 1). Eight subjects were successfully contacted, met criteria for inclusion and consented for the study. Seven subjects were able to complete the follow-up scan and repeat symptomatology scales. Compared with subjects that successfully completed the follow-up study, subjects that completed the first scan but were unable to complete the follow-up study did not differ significantly in their distribution of symptoms at the time of the first scan: Completer YMRS $22.1 \pm 8.2$ versus non-completer YMRS $27 \pm 9.3(P=0.31)$, completer MADRS $12.7 \pm 3$ versus non-completer MADRS $9.6 \pm 3.6(P=0.095)$, completer PANSS: $56.3 \pm 13$ versus non-completer PANSS $62.5 \pm 14.9(P=0.41)$.

Among the bipolar subjects that completed both manic and euthymic scans, no patients met DSM-IV criteria for panic
Table 1 Subject demographics and clinical information

\begin{tabular}{lccc}
\hline & $\begin{array}{c}\text { Healthy } \\
\text { control } \\
(\mathbf{N}=\mathbf{6})\end{array}$ & $\begin{array}{c}\text { Bipolar } \\
\text { manic } \\
(\mathbf{N}=\mathbf{7})\end{array}$ & $\begin{array}{c}\text { Bipolar } \\
\text { euthymic } \\
(\mathbf{N}=\mathbf{7})\end{array}$ \\
\hline Age & $35.2 \pm 8.1$ & $37.6 \pm 10.7$ & $39.7 \pm 10.9$ \\
Gender & $3 \mathrm{M}, 3 \mathrm{~F}$ & $3 \mathrm{M}, 4 \mathrm{~F}$ & $3 \mathrm{M}, 4 \mathrm{~F}$ \\
MADRS & - & $12.7 \pm 2.9$ & $3.71 \pm 2.7$ \\
YMRS & - & $22.1 \pm 8.2$ & $2.0 \pm 3.2$ \\
PANSS & - & $56.3 \pm 13.0$ & $35.4 \pm 3.7$ \\
Anticonvulsants & - & 5 & 3 \\
SGAs & - & 7 & 5 \\
Lithium & - & 5 & 4 \\
CPZ equivalents & - & $343 \pm 154$ & $352.2 \pm 437$ \\
Benzodiazepines & - & 5 & 2
\end{tabular}

Abbreviations: CPZ, chlorpromazine; F, female; M, male; MADRS, Montgomery-Asberg Depression Rating Scale; PANSS, Positive and Negative Syndrome Scale; SGA, second generation antipsychotic, YMRS, Young Mania Rating Scale.

Mean \pm s.d. where appropriate.

Numbers in medication rows are the number of subjects whose regimen includes that class of psychotropic medication. In addition to psychotropic medications, three bipolar subjects were prescribed other medications including one patient taking anti-hypertensive medication, one subject taking a statin, anti-hypertensive medication and oral anti-hyperglycemic medication and one subject taking a statin and a oral anti-hyperglycemic medication.

disorder, a diagnosis with known trait differences in brain lactate responsivity to chemical challenges. ${ }^{11}$ Two subjects met DSM-IV criteria for prior diagnosis of $\mathrm{EtOH}$ abuse. In neither case did the subject meet criteria within the 5 years before entering the study and neither engaged in daily $\mathrm{EtOH}$ use at any point. One of these two subjects also endorsed cannabis use that met DSM criteria for abuse over a period of 2 weeks a decade before entering the study. No other subjects described substance use that met criteria for abuse or dependence at any point currently or historically.

Magnetic resonance imaging/MRS scans. Proton MRS acquisitions were conducted on a 4 Tesla full body magnetic resonance scanner (Varian/Unitylnova, Varian, Palo Alto, CA, USA), using a 16-rung, single-tuned, volumetric birdcage coil (Robarts Research Institute, London, Ontario, Canada). Two-dimensional (2-D) gradient-recalled echo images (12s) were acquired in three planes to ensure optimal patient positioning. Manual global shimming of unsuppressed water signal yielded an unfiltered global water linewidth of $\leqslant 30 \mathrm{~Hz}$. T1-weighted sagittal images (TE/TR $=6.2 / 11.4 \mathrm{~ms}, \mathrm{FOV}=24$ $\times 24 \times 8 \mathrm{~cm}^{3}$, in-plane resolution $=0.94 \times 1.88 \mathrm{~mm}^{2}$, slice thickness $=5 \mathrm{~mm}$, readout points $=512$, flip angle $=11^{\circ}$ ) and axial images (similar parameters as above, except for in-plane resolution $=0.94 \times 0.94 \mathrm{~mm}^{2}$, slice thickness $=2.5$ $\mathrm{mm}$ ) were next acquired, allowing for clear differentiation between cortical gray matter (GM) and white matter and serving as anatomical guides to positioning MRS voxels.

A $2 \times 2 \times 2 \mathrm{~cm}^{3}$ single voxel was then placed on the anterior cingulate cortex. We placed the voxel on midsagittal T1weighted images, anterior to the genu of the corpus callosum, with the ventral edge aligned with the dorsal corner of the genu (Figure 1), and positioned the voxel on the midline on axial images. We used a point-resolved spectroscopy sequence modified for J-resolved proton $\left({ }^{1} \mathrm{H}\right)$ MRS employing a fourpulse water suppression enhanced through T1 effects (WET) sequence. ${ }^{12}$ 


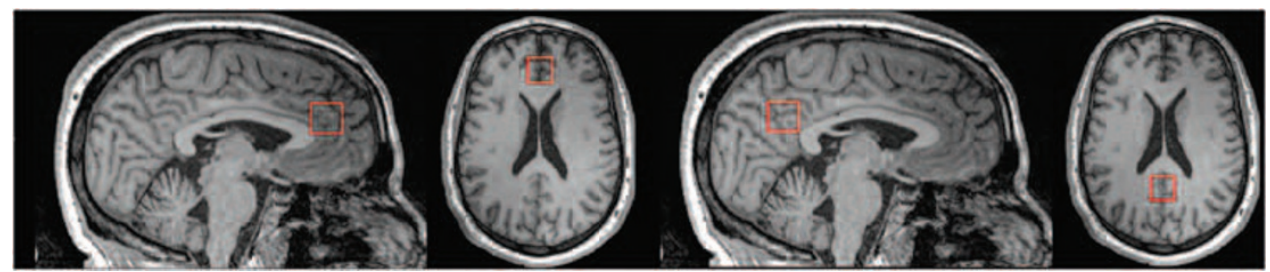

Figure 1 Placement of the anterior cingulate cortex (ACC) (left) and parietal occipital cortex (POC) (right) voxels.

Manual shimming within the voxel resulted in water linewidths $\leqslant 11 \mathrm{~Hz}$. Following flip angle, water suppression, and RF pulse power optimization using automated methods, the 2-D J-resolved sequence collected 48 TE-stepped spectra from the voxel, with the echo time ranging from 30 to $500 \mathrm{~ms}$ in 10-ms increments. Acquisition parameters were $\mathrm{TR}=2.0 \mathrm{~s}$, acquisition bandwidth $=2 \mathrm{kHz}$, repetitions $=16$, nominal voxel volume $=8 \mathrm{cc}$ and approximate scan duration $=28 \mathrm{~min}$.

This process was repeated on a $2 \times 2 \times 2-\mathrm{cm}^{3}$ parietooccipital cortex voxel. On midsagittal images, we aligned the ventral edge of the voxel with the dorsal corner of the splenium of the corpus callosum, covering posterior cingulate and retrosplenial cortices, and positioned the voxel on the midline in axial images (Figure 1). As POC spectral quality was superior, eight repetitions were obtained in $\sim 14 \mathrm{~min}$. Total time in the magnet was $75-90 \mathrm{~min}$.

MRS data processing and analysis. All MRS processing was performed blind to diagnosis. Real (not magnitude) spectra were used (Figure 2). For each voxel, the 24 TE-stepped free-induction decays were zero-filled to 128 free-induction decay in $\mathrm{f} 1$. We used the commercial spectral fitting package LC Model (version 6.1-4E, Oakville, Ontario, Canada) to fit every J-resolved spectral extraction within a bandwidth of $50 \mathrm{~Hz}(-25$ to $+25 \mathrm{~Hz})$. We used GAMMA to generate sets of 48 point-resolved spectroscopy sequence, TE-stepped spectra ranging from 30 to $500 \mathrm{~ms}$ in $10-\mathrm{ms}$ increments. For every metabolite, we fit its spectrum over $50 \mathrm{~Hz}$ bandwidth ( -25 to $+25 \mathrm{~Hz}$; 64 TE points), deriving the integrated area under the 2-D surface. ${ }^{12}$ Spectral regions outside this range did not contain significant metabolite signal but contributed additional noise. LC Model provides Cramer-Rao lower bounds (CRLBs), an estimate of the variance associated with fitting. In our case, each 2-D spectrum produced 64 CRLBs for each metabolite. These ranged from $<5 \%$ in regions of high certainty to $>50 \%$ in regions of low certainty. To accurately reflect the reliability of the 2-D-fitting procedure, we determined an average CRLB value weighted by the signal-to-noise ratio of each spectral extraction on the basis of the $\mathrm{N}$-acetylaspartate (NAA) resonance. ${ }^{9,13}$ Metabolite values with a CRLB above $25 \%$ were discarded given their poor fit.

The results of LC Model fitting for each metabolite are reported as arbitrary units. Concentrations of given metabolites are expressed as ratios of the metabolite arbitrary unit/ creatine-phosphocreatine $(\mathrm{Cr})$ arbitrary unit. No statistically significant difference in $\mathrm{Cr}$ concentration was found between $\mathrm{BD}$ subjects and HCs. ${ }^{14}$
Image segmentation. Tissue segmentation of T1-weighted images into GM, white matter and cerebrospinal fluid in each MRS voxel was calculated using Matlab (Natick, MA, USA) and the SPM toolbox (Cambridge, UK). The percentage of GM in ACC and POC was $56.0 \pm 5.7 \%$ and $49.1 \pm 7.6 \% \quad$ (BD-manic), $58.1 \pm 4.1 \%$ and $53.8 \pm 5.1 \%$ (BD-euthymic), $60.3 \pm 2.4 \%$ and $57.7 \pm 2.9 \%$ (controls) while that of white matter was $23.5 \pm 3.6 \%$ and $38.5 \pm 7.8 \%$ (BD-mania), $20.5 \pm 4.1 \%$ and $35.7 \pm 5 \%$ (BD-euthymia), $22.0 \pm 6.5 \%$ and $30.6 \pm 3.0 \%$ (controls), respectively. There was no statistically significant difference in GM\% between the bipolar patients in the euthymic state and in the manic state in the ACC $(P=0.44)$ or POC $(P=0.19)$. Nor was there any statistically significant difference in GM between the bipolar patients in the euthymic state and $\mathrm{HC}$ subjects in the ACC $(P=0.26)$ or POC $(P=0.17)$.

Statistical analysis. Given the prior literature on metabolic dysfunction in bipolar disorder and specific findings on lactate, ${ }^{4}$ glutamine/glutamate ratio ${ }^{4,9,13}$ and $\mathrm{NAA}^{15}$ in manic or mixed states, we assessed the effect of mood state (mania versus euthymia) and voxel location (ACC vs POC) on glutamine/glutamate, NAA/Cr and lactate/Cr measures using a General Linear Model analysis fit using the Mixed routine in SPSS statistical software (SPSS, Chicago, IL, USA.) with age, gender and $\mathrm{GM} \%$ as covariates. Main effects and voxel location by mood state interaction were explored. Significance was fixed at $\alpha=0.05$. We examined the effects of gender and medication (that is, medicated status versus unmedicated status for benzodiazepines, for lithium, and for anticonvulsants) on metabolite ratios using one-way analysis of variances. We calculated correlation coefficients for the relationships between age, voxel $\mathrm{GM} \%$ and $\mathrm{CPZ}$ equivalents with metabolite/Cr values. Finally, we examined MADRS, YMRS and PANSS score correlations with metabolite/Cr values.

\section{Results}

For Lactate/Cr there was a main effect of clinical state $[\mathrm{F}(1,5)=33.296, P=0.002]$ but no main effect of voxel location $[F(1,5)=0.908, P=0.383]$. When a voxel location $\times$ clinical state interaction was incorporated into the model, there was a main effect of clinical state $[F(1,6)=8.972$, $P=0.026]$ but no main effect of voxel location $[\mathrm{F}(1,6)=0.08$, $P=0.785]$ nor a voxel location $\times$ clinical state interaction $[\mathrm{F}(1,6)=0.001, P=0.981]$. Specifically, $\mathrm{Lac} / \mathrm{Cr}$ values were $36 \%$ lower in the euthymic state than in the manic state (Figure 3). 

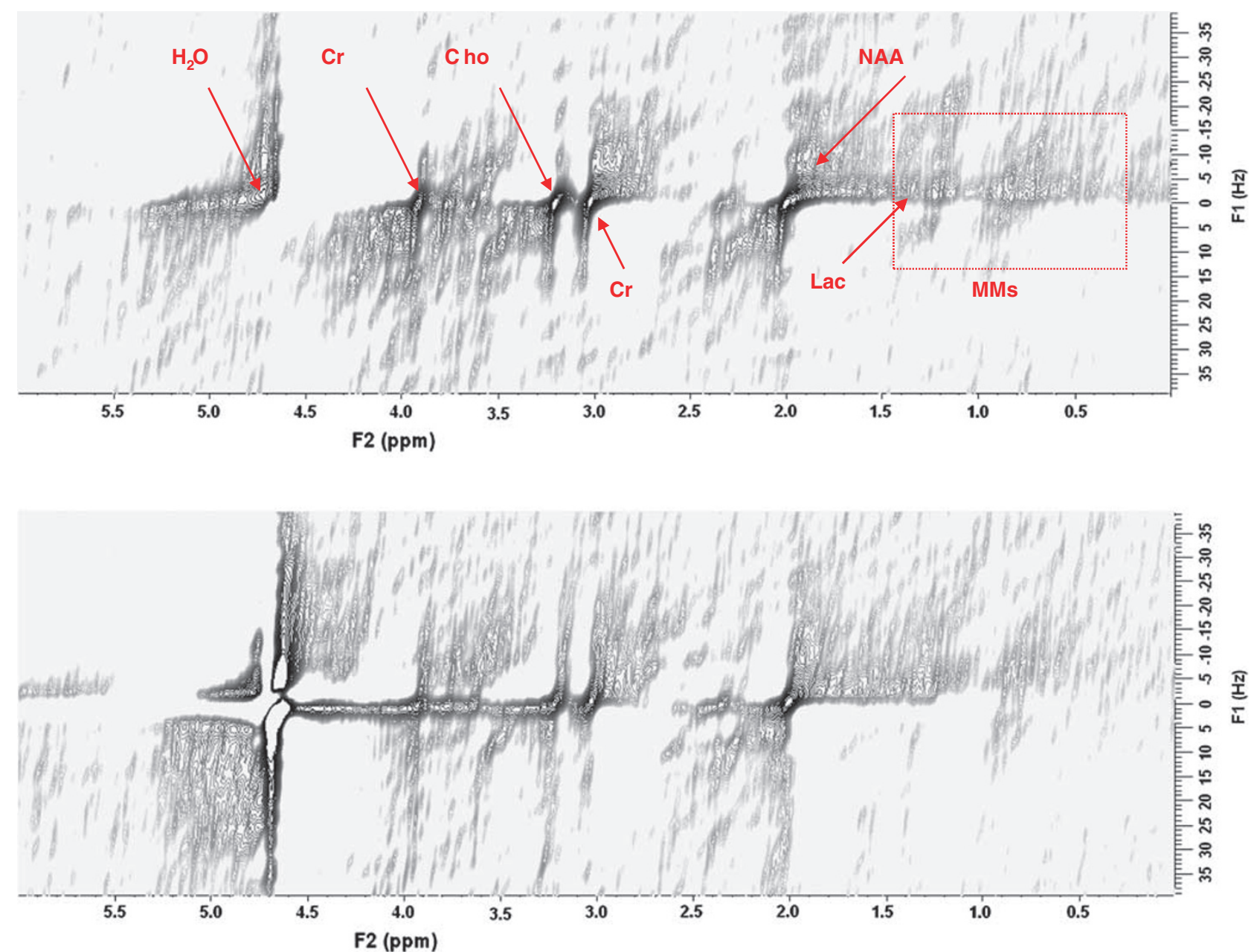

Figure 2 Contour plots of real two-dimensional (2-D) spectra from the anterior cingulate cortex (ACC) in a control (top) and bipolar disorder (BD) subject (bottom). In each case, the $X$ axis is frequency ( $F 2$ in p.p.m.) and the $Y$ axis is $\mathrm{J}(\mathrm{F} 1 \mathrm{in} \mathrm{Hz}$ ). The spectral region from about -35 to $+35 \mathrm{~Hz}$ is shown. The main metabolite resonances recognizable in the plots are labeled. Although the lactate resonance is not well-resolved in these plots, its approximate location at 1.33 p.p.m. is highlighted. The additional information available from 2-D magnetic resonance spectroscopy (MRS) allows improved fitting of this metabolite as discussed in the text. Note the variable nature of water suppression, and of the macromolecule signal profile (highlighted in a box in the top panel) in the two spectra. Lac, lactate; $\mathrm{H}_{2} \mathrm{O}$, water; GSH, glutathione; MMs, macromolecules.

For the Gln/Glu ratio there was a main effect of clinical state $[F(1,3)=10.923, P=0.043]$ but no main effect of voxel location $[\mathrm{F}(1,4)=0.067, P=0.808]$. When a voxel location $\times$ clinical state interaction was incorporated into the model, no significant differences were seen in Gln/Glu ratio between clinical states $[F(1,3)=2.369, P=0.225]$, nor by voxel location $[F(1,4)=0.365, P=0.578]$ nor was there a voxel location $\times$ clinical state interaction $[F(1,1)=0.465, P=0.615]$.

For NAA/Cr there was no main effect of clinical state $[F(1,6)=4.18, P=0.090]$. There was a significant effect of voxel location with higher NAA levels found in the POC versus ACC $[F(1,6)=76.298, P<0.001]$. When a voxel location $\times$ clinical state interaction was incorporated into the model, no significant differences were seen in NAA for clinical state $[F(1,6)=2.23$, $P=0.188]$ nor was there a voxel location $\times$ clinical state interaction $[F(1,5)=3.061, P=0.144]$. There remained a significant effect of voxel location $[F(1,5)=70.823, P<0.001]$.

One-way analysis of variance analysis revealed no statistically significant effects on lactate/Cr values by gender or the use of antipsychotics, benzodiazepines, lithium or anticonvulsants. Age, voxel GM\% and CPZ equivalents were not correlated with metabolite/ $\mathrm{Cr}$ values. YMRS, MADRS and PANSS scores did not correlate with metabolite/Cr values.

The brain lactate/ $\mathrm{Cr}$ values for patients in the manic state (mean $0.074 \pm 0.040$ ) were similar to those of a set of age- and gender-matched control subjects (mean $0.070 \pm 0.028$ ) but significantly $(P=0.049)$ lower in patients in the euthymic state (mean $0.048 \pm 0.016$ ) compared with controls (Figure 3 ).

\section{Discussion}

To our knowledge this is the first longitudinal ${ }^{1} \mathrm{H}$-MRS study across mania and euthymia of patients diagnosed with bipolar disorder type I. Patients diagnosed with bipolar disorder type I demonstrated a state related change in brain lactate concentration with euthymic subjects showing a significantly lower lactate signal compared with the same subjects in a manic state. When compared with age- and gender-matched 


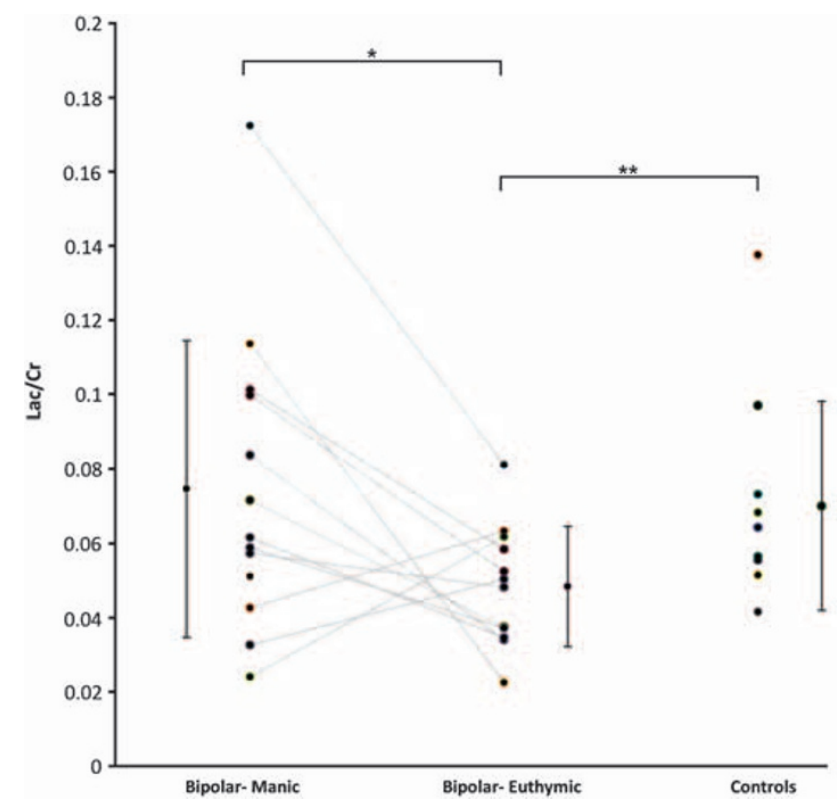

Figure 3 Brain lactate concentrations measured as lactate/creatinine ratios in the parietal occipital cortex (POC) and anterior cingulate cortex (ACC) for bipolar patients in a manic state (left), bipolar patients in a euthymic state (middle), and healthy control subjects (right). Identical voxel locations in the same subjects are connected by gray lines. The group mean (error bars $=$ s.d.) is shown at the side for each time point. ${ }^{*} P=0.002 ;{ }^{* *} P=0.049$.

HCs, bipolar disorder patients in the manic state showed no abnormality in brain lactate concentration. Euthymic bipolar disorder patients demonstrated a significantly decreased lactate concentration when compared with a $\mathrm{HC}$ population.

The observation that lactate is nearly normal in the manic state and significantly reduced in euthymia is intriguing. Prior observations in ${ }^{31} \mathrm{P}$ MRS suggest a similar trajectory of the membrane precursor phosphomonoester (PME) peak between pathological mood states and euthymia. Kato et al. ${ }^{3}$ demonstrated that PME was modestly increased in mania versus control subjects and that PME decreased to a significantly lower than control level in euthymia. A study by Kato et al. ${ }^{16}$ and a meta-analysis conducted by Yildiz et al. ${ }^{17}$ found a similar trajectory in which bipolar patients in the depressed state have elevated PME while those in a euthymic state have PME levels decreased compared with controls. A recent study by $\mathrm{Kim}$ et al. ${ }^{18}$ of quetiapine in the treatment of rapid-cycling bipolar disorder demonstrated a significant decrease in brain lactate during treatment with quetiapine. That study could not differentiate metabolic changes due to quetiapine administration versus changes because of improvement in clinical state and subjects were not followed to euthymia.

What might be the physiological significance of the observed lactate changes? In synthesizing the growing body of findings about metabolic dysfunction in bipolar disorder, Stork et al. ${ }^{1}$ proposed that mitochondrial dysfunction causing impaired oxidative phosphorylation is a hallmark of bipolar disorder. In this model, mitochondrial dysfunction results in a shift to anaerobic metabolism and increased lactate observed in bipolar disorder in a depressed or mixed-state. ${ }^{4}$ This mitochondrial dysfunction also may result in alterations in PME levels ${ }^{3,17}$ thought to represent changes in phospholipid membrane metabolism. Kato and Kato ${ }^{19}$ hypothesized that bipolar disorder is characterized by a trait metabolic dysfunction that is demonstrated in the abnormal findings observed in euthymia. They conjectured that the brain's attempts to 'correct' these abnormalities through increased monoaminergic neurotransmission might induce pathological mood states such as depression or mania. In other words, neither mood episodes nor euthymia are truly healthy states in bipolar disorder. Rather, they represent varying degrees of compensation to underlying abnormalities.

In examining the effect of clinical state on other metabolites it is worth noting that the GIn/Glu ratio demonstrated a significant $(P=0.046)$ effect of clinical state. This finding did not meet criteria for significance when corrected for multiple comparisons (adjusted $\alpha=0.016$ ), nor was there a significant effect of clinical state when state by anatomical region interaction was incorporated into the model. Nevertheless, this result suggests that $\mathrm{G} / \mathrm{n} / \mathrm{Glu}$ ratio abnormalities previously observed in bipolar disorder ${ }^{9}$ may in fact vary with clinical state. This finding bears further investigation.

The strengths of this study include the investigation of inpatients who are highly resistant to engage in research studies, the longitudinal design and the assessment of both euthymic and manic states in the same individuals, a challenging task. ${ }^{20}$ All of our subjects were scanned on the same high field (4T) magnet at both time points and we have included a control population. Medication regimens were relatively consistent at both time points.

One limitation of our study is a small sample size that makes it difficult to rule out type I errors. This applies both to patients as well as $\mathrm{HC}$ subjects whose small numbers represent an attempt to provide a match for age and gender to the patient population. Another limitation is the generalizability of these findings. Although manic state scans were all acquired in a population symptomatic enough to require inpatient hospitalization, the patients who were able to complete the manic state scan were generally less symptomatic than those who could not complete the scan. Additionally, at the time of the euthymic state, all subjects were in ongoing psychiatric treatment, free of substance abuse or dependence and were able to be contacted and perform the follow-up study as outpatients. It is possible that these patients may have better inter-episode functioning and better prognosis thereby limiting generalization to the bipolar disorder population as a whole. Finally, although we cannot rule out an effect of medications on our findings, the longitudinal nature of this experiment was designed to minimize differences in medication regimens at both time points.

This demonstration of a relationship between metabolic dysfunction and mood state has implications for both the diagnosis of bipolar disorder as well as its treatment. Epidemiological studies demonstrate that at least $20 \%$ of patients hospitalized for unipolar depression will go on to have a diagnosis of bipolar disorder type . $^{21}$ The development of a biomarker measurable at hospitalization that can differentiate euthymia in bipolar disorder versus unipolar depression would be of significant diagnostic and predictive value. Similar studies are needed to compare brain lactate in both bipolar disorder as well as unipolar depression across depressive and euthymic states. 
The demonstration of a biomarker intimately related to clinical state will possibly allow evaluation of novel therapies. Notably, Kim et al. ${ }^{18}$ have already performed a study examining both symptomatology score changes we well as changes in lactate in response to quetiapine. Although Kim et al. did not follow patients to euthymia; they did note a significant association between change in lactate level and clinical response. This finding may be extended to other interventions and may allow for the early differentiation of patients who will achieve response from those who will remain symptomatic.

\section{Conflict of interest}

Dr Renshaw is a consultant to Novartis, GlaxoSmithKline and Kyowa Hakko. Dr Öngür is PI on a research contract with Rules Based Medicine Inc. The other authors declare no conflict of interest.

Acknowledgements. We would like to thank the individuals who participated in this study. We thank Caitlin Ravichandran and Harvard Catalyst for help with statistical analyses. We thank Roscoe Brady Sr for helpful discussions and comments on the manuscript. This work was supported by the Dupont-Warren Fellowship from the Department of Psychiatry of Harvard Medical School to RB, NIMH award K23MH079982 to DO, a philanthropic grant from the Taplin Foundation to DO and BC, and by funds provided by The Department of Psychiatry, Beth-Israel Deaconess Medical Center to MK. Dr Renshaw has received research support from Roche, GlaxoSmithKline and Eli Lilly.

1. Stork C, Renshaw PF. Mitochondrial dysfunction in bipolar disorder: evidence from magnetic resonance spectroscopy research. Mol Psychiatry 2005; 10: 900-919.

2. Dager SR, Corrigan NM, Richards TL, Posse S. Research applications of magnetic resonance spectroscopy to investigate psychiatric disorders. Top Magn Reson Imaging 2008; 19: 81-96

3. Kato T, Takahashi S, Shioiri T, Inubushi T. Alterations in brain phosphorous metabolism in bipolar disorder detected by in vivo $31 \mathrm{P}$ and $7 \mathrm{Li}$ magnetic resonance spectroscopy. J Affect Disord 1993; 27: 53-59.

4. Dager SR, Friedman SD, Parow A, Demopulos C, Stoll AL, Lyoo IK et al. Brain metabolic alterations in medication-free patients with bipolar disorder. Arch Gen Psychiatry 2004; 61: 450-458.

5. Cataldo AM, McPhie DL, Lange NT, Punzell S, Elmiligy S, Ye NZ et al. Abnormalities in mitochondrial structure in cells from patients with bipolar disorder. Am J Pathol 2010; 177: 575-585.
6. Maj M, Pirozzi R, Starace F. Previous pattern of course of the illness as a predictor of response to lithium prophylaxis in bipolar patients. J Affect Disord 1989; 17: 237-241.

7. Tohen M, Waternaux CM, Tsuang MT. Outcome in Mania. A 4-year prospective follow-up of 75 patients utilizing survival analysis. Arch Gen Psychiatry 1990; 47: 1106-1111.

8. Fountoulakis KN, Giannakopoulos P, Kovari E, Bouras C. Assessing the role of cingulate cortex in bipolar disorder: neuropathological, structural and functional imaging data. Brain Res Rev 2008; 59: 9-21.

9. Ongur D, Jensen JE, Prescot AP, Stork C, Lundy M, Cohen BM et al. Abnormal glutamatergic neurotransmission and neuronal-glial interactions in acute mania. Biol Psychiatry 2008; 64: 718-726.

10. First MB. New York State Psychiatric Institute. Biometrics Research D. Structured Clinical Interview for DSM-IV-TR Axis I Disorders: SCID-I. Biometrics Research Dept., New York State Psychiatric Institutute: New York, NY, 2007.

11. Maddock RJ, Buonocore MH, Copeland LE, Richards AL. Elevated brain lactate responses to neural activation in panic disorder: a dynamic 1H-MRS study. Mol Psychiatry 2009; 14: $537-545$.

12. Jensen JE, Licata SC, Ongur D, Friedman SD, Prescot AP, Henry ME et al. Quantification of J-resolved proton spectra in two-dimensions with LCModel using GAMMA-simulated basis sets at 4 Tesla. NMR Biomed 2009; 22: 762-769.

13. Michael N, Erfurth A, Ohrmann P, Gossling M, Arolt V, Heindel W et al. Acute mania is accompanied by elevated glutamate/glutamine levels within the left dorsolateral prefrontal cortex. Psychopharmacology 2003; 168: 344-346.

14. Ongur D, Prescot AP, Jensen JE, Cohen BM, Renshaw PF. Creatine abnormalities in schizophrenia and bipolar disorder. Psychiatry Res 2009; 172: 44-48.

15. Cecil KM, DelBello MP, Morey R, Strakowski SM. Frontal lobe differences in bipolar disorder as determined by proton MR spectroscopy. Bipolar Disord 2002; 4: 357-365.

16. Kato T, Takahashi S, Shioiri T, Inubushi T. Brain phosphorous metabolism in depressive disorders detected by phosphorus-31 magnetic resonance spectroscopy. J Affect Disord 1992; 26: 223-230.

17. Yildiz A, Sachs GS, Dorer DJ, Renshaw PF. 31P Nuclear magnetic resonance spectroscopy findings in bipolar illness: a meta-analysis. Psychiatry Res 2001; 106: $181-191$.

18. Kim DJ, Lyoo IK, Yoon SJ, Choi T, Lee B, Kim JE et al. Clinical response of quetiapine in rapid cycling manic bipolar patients and lactate level changes in proton magnetic resonance spectroscopy. Prog Neuropsychopharmacol Biol Psychiatry 2007; 31: $1182-1188$.

19. Kato T, Kato N. Mitochondrial dysfunction in bipolar disorder. Bipolar Disord 2000 2(3 Part 1): 180-190.

20. Joffe RT, MacQueen GM, Marriott M, Trevor Young L. A prospective, longitudinal study of percentage of time spent ill in patients with bipolar I or bipolar II disorders. Bipolar Disord 2004; 6: 62-66.

21. Goldberg JF, Harrow M, Whiteside JE. Risk for bipolar illness in patients initially hospitalized for unipolar depression. Am J Psychiatry 2001; 158: 1265-1270.

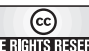

Translational Psychiatry is an open-access journal published by Nature Publishing Group. This work is licensed under the Creative Commons Attribution-NonCommercial-No Derivative Works 3.0 Unported License. To view a copy of this license, visit http://creativecommons.org/licenses/by-nc-nd/3.0/ 